\title{
New Approaches to Lunar Ice Detection and Mapping
}

\author{
The Scientific Importance of the Moon's Polar Ice Deposits
}

\section{Authors:}

Paul O. Hayne ${ }^{1}$, David A. Paige ${ }^{2}$, Andrew P. Ingersoll ${ }^{3}$, Oded Aharonson ${ }^{4}$, Shane Byrne ${ }^{5}$, Barbara Cohen $^{6}$, Anthony Colaprete ${ }^{7}$, Christopher S. Edwards ${ }^{8}$, Emily J. Foote ${ }^{2}$, Benjamin T. Greenhagen ${ }^{9}$, Amanda Hendrix ${ }^{10}$, Brendan Hermalyn ${ }^{11}$, Mihaly Horanyi ${ }^{1}$, Yang Liu ${ }^{12}$, Paul G. Lucey $^{11}$, Benjamin K. Malphrus ${ }^{13}$, Thomas B. McCord ${ }^{14}$, Michael J. Poston ${ }^{15}$, Gerald B. Sanders ${ }^{16}$, Norbert Schörghofer ${ }^{10}$, Matthew M. Siegler ${ }^{10,17}$, Robert L. Staehle ${ }^{12}$, Wayne Zimmerman ${ }^{12}$, and the members of the study sponsored by the Keck Institute for Space Studies

\footnotetext{
${ }^{1}$ University of Colorado Boulder

${ }^{2} \mathrm{UCLA}$

${ }^{3}$ California Institute of Technology

${ }^{4}$ Weizmann Institute of Science

${ }^{5}$ University of Arizona

${ }^{6}$ NASA - Goddard Space Flight Center

${ }^{7}$ NASA - Ames Research Center

${ }^{8}$ Northern Arizona University

${ }^{9} \mathrm{JHU} / \mathrm{APL}$

${ }^{10}$ Planetary Science Institute

${ }^{11}$ University of Hawai'i

${ }^{12}$ NASA - Jet Propulsion Laboratory

${ }^{13}$ Morehead State University

${ }^{14}$ Bear Fight Institute

${ }^{15}$ Southwest Research Institute

${ }^{16}$ NASA - Johnson Space Center

${ }^{17}$ Southern Methodist University
} 


\section{Overview}

Water and other volatiles are fundamental tracers of habitable environments on terrestrial planets and satellites. Yet after decades of exploration, the origins, abundances, and distributions of volatiles and their effects on planetary evolution are not fully understood. The discovery of substantial water ice deposits in the polar regions of both Mercury and the Moon presents an opportunity to test hypotheses regarding the delivery and retention of water and other volatiles in the inner solar system. As Earth's closest planetary neighbor, the Moon thus is thus a keystone for addressing outstanding problems in planetary science directly linked to planetary origins and habitability. Furthermore, water on the Moon is of great interest to the exploration community, as a resource for astronauts and robotic missions of the future. However, as we summarize below, major outstanding questions remain regarding the distribution, abundance, and origins of lunar water, which must be answered in order to advance this important field of study.

This paper summarizes the consensus science priorities established by a group of $>30$ experts in lunar and planetary science regarding the investigation of the Moon's polar ice deposits based on a Keck Institute for Space Studies (KISS) study (Hayne et al., 2014). We developed two fundamental, interrelated questions that can be answered in the next decade through lunar polar exploration:

1) What are the origins and evolution of water in the inner solar system?

2) Where are the operationally useful deposits of water on the Moon?

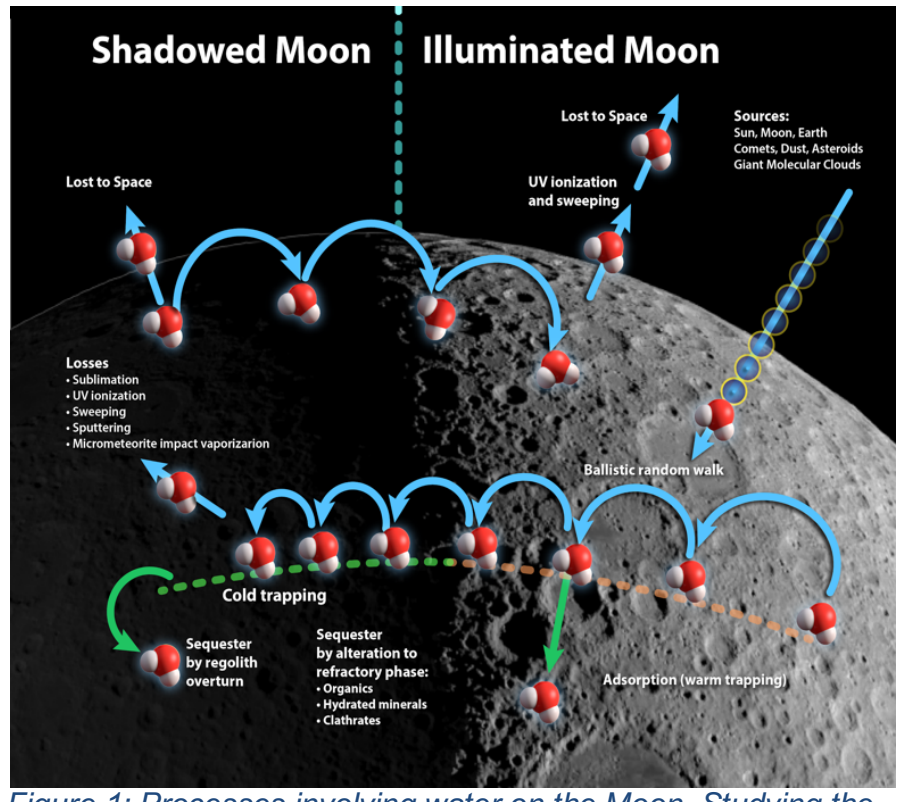

Figure 1: Processes involving water on the Moon. Studying the "lunar water cycle" can provide critical understanding of similar processes operating throughout the solar system. (From: Hayne et al., 2014; adapted from Lucey et al., 2009)
In Section 2, we explain why lunar ice elicits such excitement in the planetary science community, and should be among the highest-priority targets of study for the next decade. Answering the two questions above will benefit from a common set of measurements, as detailed in Section 3. In the remainder of the paper, we outline a path towards achieving the goals promoted here. Since this path can be very effectively implemented through a program of small missions, we strongly recommend NASA continue to support low-cost, innovative missions and instrument development through programs such as SIMPLEx.

\section{Scientific Context and Importance of Lunar Ice}


Water is chief among the volatiles in terms of its effects on planetary interior and surface evolution, as well as habitability (e.g., Phillips et al., 2001; Ruedas et al., 2013; Seager et al., 2013). The present-day distribution of water in the inner solar system depends on the initial conditions in the protoplanetary disk, as well as the past and ongoing delivery of asteroidal and cometary material scattered by dynamical instabilities (O'Brien et al., 2014). In the early 1960's Watson, Murray, and Brown (1961, 1962; collectively "WMB") recognized that the lunar poles should harbor permanently shadowed regions (PSRs) cold enough to potentially trap water and other volatiles for $>1$ Gyr. If these cold traps exist, they argued, a pristine record of water delivery to the Earth-moon system could be accessed relatively easily. NASA began sponsoring limited studies into the possible exploration for and utilization of lunar volatiles resources in the early 1980s (Steurer et al., 1983; Carroll et al, 1983; Staehle, 1983). An obscure publication of Robert Goddard's notes indicated that he had given serious thought to the possibility of the lunar poles being a cold trap for ice as early as 1920 (Goddard 1920).

Since WMB's initial predictions, numerous theoretical and observational studies have bolstered the case for lunar polar ice deposits and further explored their intricate, patchwork history and significance (e.g., see the excellent review by Lawrence (2017), as well as the report by Hayne et al. (2014) on which this white paper is based). To summarize current understanding:

1) There is ice at the lunar poles, with $\mathrm{H}_{2} \mathrm{O}$ abundances locally up to at least 10 wt\% (Colaprete et al., 2010; Li et al., 2018)

2) Lunar polar volatiles are distributed heterogeneously within the PSRs (Hurley et al., 2012; Hayne et al., 2015)

3) Temperatures within the Moon's PSRs are among the coldest in the solar system and capable of trapping a wide range of comet-like volatiles (Paige et al., 2010; Williams et al., 2019)

In addition to the polar ice deposits, widespread hydration has been measured on the lunar surface and near subsurface using multiple remote sensing techniques (Feldman et al., 2001; Clark et al., 2009; Pieters et al., 2009; Sunshine et al., 2009; Hendrix et al., 2012). Some of these observations also implied active exchange with the regolith and potential transport through the exosphere (e.g., Hendrix et al., 2019), consistent with lab measurements, although inconsistencies currently persist between observations and theoretical models (Schörghofer, 2014). The remote sensing observations are consistent with state-of-the-art laboratory analyses of returned lunar samples, which show $\sim 100-1000$ ppm of $\mathrm{H}_{2} \mathrm{O}$ within pristine mineral grains such as apatites (Saal et al., 2008; Liu et al., 2012; Boyce et al., 2014). Furthermore, it is now widely recognized that $\mathrm{H}_{2} \mathrm{O}$ on the Moon is part of an interconnected system, which can be used to study phenomena such as space weathering, atmospheric escape, micrometeorite bombardment, and more (Figure 1).

Detecting and mapping water and other volatiles is critical to testing hypotheses regarding their origins and the processes affecting their movement, loss, and sequestration at the poles. For example, a primarily cometary source would provide a local, punctuated influx of volatiles (Ong et al., 2010), with a compositional signature (Colaprete et al., 2010) distinct from 
that of lunar outgassing (Needham and Kring, 2017) or solar wind $\mathrm{H}$-implantation (Li and Millikan, 2017). In addition, D/H ratios should be preserved during transit to the Moon's polar cold traps (Schörghofer, 2014), providing a potentially diagnostic signature of the source materials. The ages of the ice deposits may also be inferred from the depth of regolith cover, vertical and horizontal heterogeneity (Hurley et al., 2012; Rubanenko et al., 2019) or geologic mapping (Deutsch et al. 2020a,b). Photodestruction and burial are sufficiently rapid that detection of widespread ice near the surface would imply a rapid influx to the Earth-Moon system in the geologically recent past (Morgan and Shemansky, 1991; Farrell et al., 2019).

\section{Outstanding Questions and Key Measurements Needed}

Existing measurements provide an incomplete picture of lunar polar volatiles. For example, the Lunar CRater Observation and Sensing Satellite (LCROSS) mission was successful at detecting $\sim 5-10$ wt\% $\mathrm{H}_{2} \mathrm{O}$ at a single location within a PSR in Cabeus crater near the lunar south pole (Colaprete et al., 2010). However, this location was chosen precisely because it was likely to yield a positive result, as one of the best cold traps on the Moon (Figure 2): very cold temperatures < $90 \mathrm{~K}$ (Hayne et al., 2010; Paige et al., 2010), high concentrations of hydrogen (Boynton et al., 2011), as well as hints of ice-like radar reflections revealed through later observations (Patterson et al., 2017). Therefore, although this successful mission provided extremely tantalizing data, LCROSS alone does not meet the definition of a controlled experiment. In fact, inconsistencies remain among various datasets regarding the distribution of water and other volatiles (e.g., Hayne et al., 2015; Lawrence et al., 2017). It is unclear whether these inconsistencies are the result of measurement uncertainty, or intrinsic variations on the Moon; it is therefore critical to make more and better measurements in order to resolve the uncertainties. We propose that an expanded number of quantitative water ice measurements at multiple locations is the best and most efficient way of accomplishing this goal.

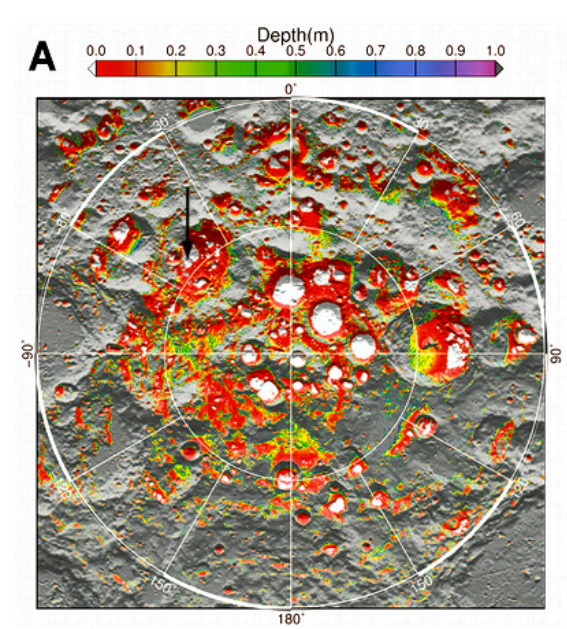

Figure 2: The south polar region of the Moon. (A) depth to ice stability (Paige et al., 2010), and (B) neutron count rate, indicating $[H]$ proportional to neutron suppression. The LCROSS impact site is indicated by the arrow in both panels.
We find that a single set of "key measurement requirements" can be used to directly address the questions highlighted above.

\section{Key Measurements:}

Determine the concentration of water in the upper few meters of lunar regolith with sensitivity better than 0.5 $w t \%$, at multiple locations with diverse thermal environments. 
Although a single mission could in principle be formulated to make these measurements, our study found that a program of multiple missions could make steady and quantifiable progress toward this goal. Here, we do not intend to recommend a specific mission or program; rather, we wish to convey the importance of detecting, quantifying, and mapping lunar polar ice deposits for the whole field of planetary science. A secondary objective is to highlight low-cost mission concepts that could accomplish the science, which are deserving of further study.

\subsection{Outstanding Questions}

Recent observations have proven that ice deposits exist on the Moon. This discovery has led to the more nuanced question of "What is the geographical extent, quantity, and origin of lunar water and other volatiles?" Although significant progress has been made, several important sub-questions remain.

\section{The inventory and distribution of volatiles is fundamental:}

1. Is there molecular water in sunlit regions of the Moon, or only hydroxyl $(\mathrm{OH})$ ? (Pieters et al., 2009; McCord et al., 2011)

2. What is the geographic distribution of subsurface water, and its vertical extent? (Hurley et al., 2012; Rubanenko et al., 2019)

3. What other volatiles are present, besides water? (Colaprete et al., 2010; Hayne et al., 2015)

The amount of water is also unknown:

1. Is surface ice present in sufficient quantities for science and in-situ resource utilization (ISRU)? (Hayne et al., 2014 define this as $>0.5 \mathrm{wt} \%$ based primarily on ISRU)

2. Do higher concentrations of ice exist at depth? (Rubanenko et al., 2019)

The form of water could tell us about its origins and history:

1. Are ice deposits concentrated in layers? (Patterson et al., 2017)

2. Does ice form monolayers (coatings) on grains or fill the voids? (Siegler et al., 2011)

3. Does ice migrate vertically in response to thermal forcings? (Schörghofer and Aharonson, 2014)

4. Do individual comet impacts form discrete ice layers? (Ong et al., 2010)

\subsection{Roadmap of Lunar Ice Investigation}

Both the science and exploration of lunar volatiles will benefit from prioritizing investigations according to obtaining the Key Measurements above (Figure 3). Once this set of measurements has been acquired, we reach a potential bifurcation between science and exploration objectives. Nonetheless, these efforts remain two sides of the same coin. For example, the major science objective of obtaining the $\mathrm{D} / \mathrm{H}$ ratio of lunar polar ice can also inform exploration through better understanding of the source(s) of volatiles, which will guide resource 
assessment and utilization (e.g., Cannon and Britt, 2020). Upcoming missions are likely to make substantial progress towards achieving the Key Measurements. We first present several approaches that have not yet been selected for flight, but which could readily close the gap, using relatively modest resources.

\section{Measurement} Approaches

\subsection{Orbiters}

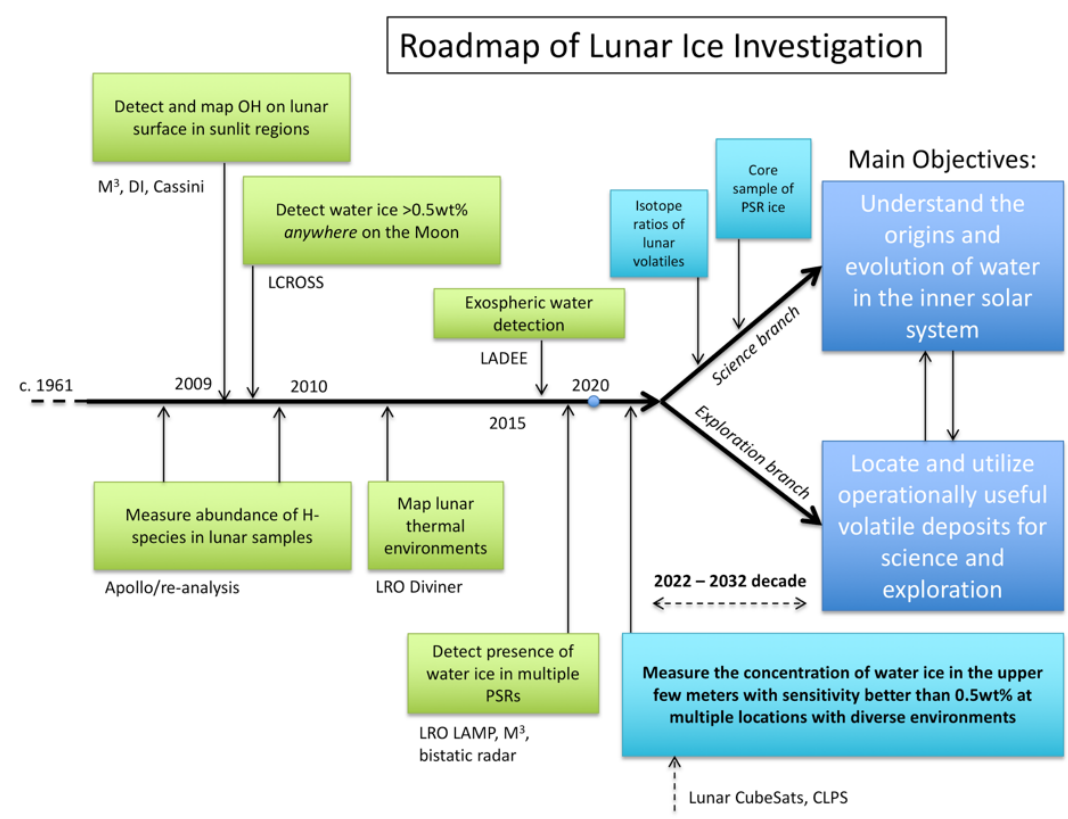

Figure 3: Proposed "roadmap" of the lunar ice investigation. The key measurements constitute a critical step in the science and exploration of lunar ice, and can be accomplished with relatively modest resources.

Innovative techniques promise to make headway towards the objective of quantifying and mapping lunar polar ice from orbit. First, active near-IR spectroscopy can provide measurements of surface composition over a range of important volatile species $\left(\mathrm{H}_{2} \mathrm{O}, \mathrm{CO}_{2}, \mathrm{CO}\right.$, $\mathrm{CH}_{4}, \mathrm{NH}_{3}$, etc) by providing the illumination source on-board the spacecraft (e.g., Vinckier et al., 2017) rather than using reflected sunlight. Passive thermal-IR spectroscopy can also utilize emission spectra to identify volatile ices on the surface, and sub-millimeter or UV spectroscopy can be used to identify water vapor released by impacts into volatile-rich surface materials.

Natural impacts into the lunar surface present a compelling opportunity to perform LCROSSstyle investigations of multiple or many locations in the lunar polar regions. Combined with passive sub-mm or UV sounding of the released vapor, icy dust grains may also be measured insitu from a polar lunar orbit. A dust instrument onboard a polar orbiting lunar spacecraft could make fundamental measurements to assess the availability and accessibility of water ice in PSRs. In the absence of an atmosphere, each incoming interplanetary dust particle directly hits the lunar surface and generates a copious number of secondary particles, with sufficient speeds, reaching over $200 \mathrm{~km}$ altitude before most of them returns to the surface. The continually present dust ejecta cloud was observed by LADEE's Lunar Dust Experiment (Horanyi et al., 2015). A more capable dust instrument like Cassini's Cosmic Dust Analyzer (Srama et al., 2005), in addition to the size and speed of an impacting particle, can also measure its composition by recording the time-of-light spectra of the ions generated when a surface sample secondary ejecta particle vaporizes and ionizes hitting the target of the detector. A modern version of CDA, the Surface Dust Analyzer (SUDA) is now being built for the Europa the Europa Clipper mission. A SUDA-type instrument in a polar orbit would generate a surface composition map, including water ice deposits in PSRs, with a spatial resolution comparable to the height of the spacecraft (Postberg et al., 2011). 
Observations of the illuminated surface have detected surface thermal emission from the 6- $\mu \mathrm{m}$ $\mathrm{H}-\mathrm{O}-\mathrm{H}$ bend of the water molecule using the SOFIA airborne observatory (Honniball et al. 2020). This method unambiguously detects molecular water resolving the issue of distinguishing hydroxyl from water that is endemic using data at $3 \mu \mathrm{m}$, and an orbital experiment would map the behavior of adsorbed water with lunar latitude and time of day, as well as detect water in key lunar geologic features. Laser spectroscopy at $3 \mu \mathrm{m}$ would also detect any variation in hydroxyl abundance on the illuminated surface without the need for thermal corrections and the associated uncertainties that have plagued passive measurements of hydration at this wavelength (Bandfield et al., 2019), and enable corroboration of the UV observations.

\subsection{Impactors}

Targeted, well-characterized impacts observed from separate Moon- or Earth-based platforms using UV to microwave spectroscopy could be used in a similar manner to LCROSS. This experiment would be performed at $\geq 3$ polar locations with distinct thermal environments (e.g., ice unstable, ground ice stable, surface ice stable). Numerical simulations (Hayne et al., 2014) indicate that very small impactors such as $\sim 1-k g$ CubeSats would produce $\sim 1000 x$ lower ejecta mass at a given altitude than LCROSS. Therefore, observations must be performed at lower altitudes and/or with greater sensitivity for these small impactors. Nonetheless, we consider this a promising approach to acquiring a scattered set of measurements for relatively low cost.

\subsection{Penetrators}

Penetrators are a class of instrumented probes capable of surviving high-speed impact into planetary surfaces and making measurements at various depths. They are therefore extremely well-suited in principle to achieving the Key Measurements outlined here. For example, we found that $\geq 3$ identical penetrators instrumented with neutron detectors, tunable laser spectrometers (TLS), and temperature probes could readily satisfy the measurement requirements. By making in-situ measurements of both hydrogen content and a detailed inventory of evolved gases, the penetrator concept could bring rich new information to bear on existing orbital remote sensing datasets.

\subsection{Landers and Rovers}

Based on the need for multiple measurements of volatile composition and abundance within locales of varying thermal environments, a single lander must be capable of remote sensing from a vantage point with access to diverse thermal environments; or, multiple landers should be used. Similarly, a rover should be capable of traversing and sampling diverse polar thermal environments. Care must be taken when interpreting results from the exposed surface of the Moon near a lander, since the landing exhaust will adsorb to the surface.

\section{Current and Planned Missions}


Missions expected to fly in the next 2-3 years will begin to address the lunar ice Key Measurements above. The last decade saw the emergence of small-sats as viable platforms for planetary science, with the Moon front and center. We strongly recommend NASA continue to support low-cost, innovative missions and instrument development through programs such as SIMPLEX.

Lunar Flashlight (Hayne et al., 2013; Cohen et al., 2017), Lunar IceCube (Clark et al., 2016), and LunaH-Map (Hardgrove et al., 2015), the three Artemis 1 lunar orbiting CubeSats will make coordinated observations of lunar volatiles in the South Polar region. As such, the three missions represent the first ad-hoc constellation of smallsats fielded to study the Moon. Lunar Trailblazer (Ehlmann et al., 2019), uses reflectance and thermal emission spectroscopy to simultaneously measure temperature and volatile composition. In addition to the low-cost small-sat missions above, NASA has also directed larger, Discovery- or New Frontiers-class missions for lunar polar volatile exploration (e.g., VIPER and CLPS 19C; Colaprete et al., 2019; Hayne et al., 2019). We note that though important scientifically, larger missions pose a potentially irreversible contamination risk to the PSRs, and a sequence of smaller, competed missions would answer the science questions through more systematic means.

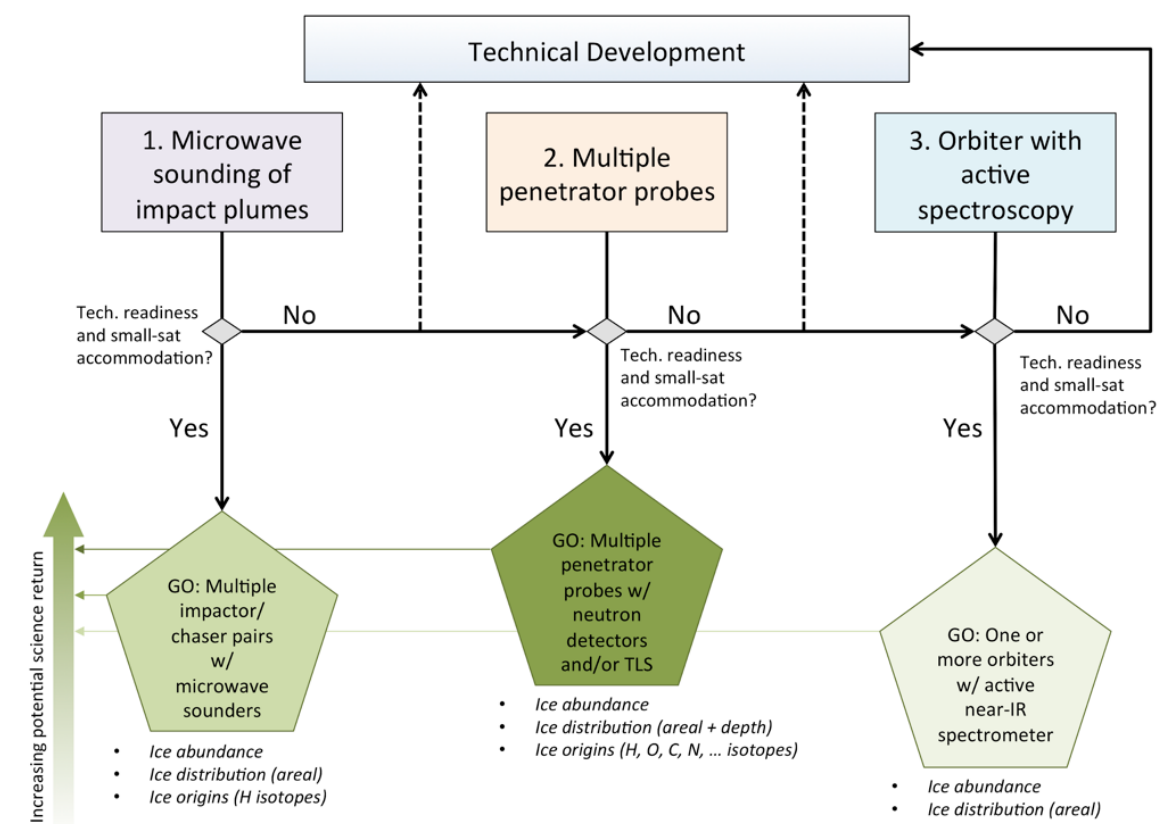

Figure 4: Decision tree for lunar volatiles investigation. (See: Hayne et al., 2014 for more information)

\section{Future Directions and Recommendations}

In addition to the recommendations already stated, we propose that maximum science return can be realized using a coordinated approach (Figure 4). The highest priority mission considered here is the penetrator concept, which can accomplish the Key Measurements most capably. However, given the anticipated technical readiness, a

multiple-impact mission could be ready to fly sooner. The flowchart shown in Fig. 4 is notional, and could be readily modified depending on technical developments in the coming decade.

\section{References:}

https://phayne.github.io/blog/2020/decadal-survey-references/ https://kiss.caltech.edu/final reports/Lunar Ice final report.pdf 